\title{
Mobilitätssuffizienz als Lösungsbeitrag zur Treibhausgasreduktion
}

\author{
Individuelle Alltagsmobilität kann auch ohne \\ Verzicht auf Aktivitäten zu deutlichen Treib- \\ hausgasreduktionen führen und zudem \\ Gewinne an Lebensqualität bringen. Dies zeigt \\ eine aktuelle Fallstudie. Doch es werden ge- \\ zielte Wirtschaftsangebote benötigt, um noch \\ mehr Suffizienz zu ermöglichen. \\ Von Markus Profijt
}

$\mathrm{L}^{2}$ aut Klimaschutzplan will die deutsche Bundesregierung die Treibhausgasemissionen bis zum Jahr 2050 um 80-95\% bezogen auf 1990 senken (BMUB 2016, S.7). Als wesentlicher Verursacher mit einem Anteil von 18,4\% emittierte der Verkehrssektor, diesem Plan konträr, im Jahr 2016 sogar zwei Millionen Tonnen Treibhausgase mehr als 1990 (UBA 2017). Da technische Errungenschaften der letzten 26 Jahre hier keine Minderung herbeiführen konnten, stellt sich die Frage, ob ein geändertes Konsumverhalten dieses erreichen kann.

Als Nachhaltigkeitsstrategie kann die Suffizienz durch verändertes oder reduziertes Konsumverhalten und einen daraus resultierenden verringerten konsuminduzierten Ressourcenund Umweltverbrauch die Schädigung der Umwelt vermindern. Als Lösungsbeitrag bisher wenig betrachtet wurde die Anwendung der Nachhaltigkeitsstrategie der Suffizienz auf das Mobilitätsverhalten. Eine Literaturrecherche ergab drei Möglichkeiten des suffizienten Mobilitätskonsums, die Inhalt der folgenden Arbeitsdefinition wurden.

Mobilitätssuffizienz ist eine Nachhaltigkeitsstrategie, die durch individuell genügsamen Mobilitätskonsum zu einer reduzierten Schädigung der Umwelt in Form von Ressourcenund Energieverbrauch führt. Dafür stehen drei Handlungsoptionen zur Verfügung:

I Wege mit verhaltensbedingt geringerem Emissionsfaktor zurücklegen,

I Wegelänge verkürzen,

I Wegeanzahl verringern.

\section{Quantitative und qualitative Fallstudie}

Mit einer gemischt quantitativen und qualitativen explorativen empirischen Forschung mit Fragebogen, Wegetagebuch und Interview wurden das spezifische Mobilitätsverhalten und der Bedarf suffizienzorientierter Konsument/innen an für sie hilfreicher Infrastruktur und zusätzlichen Mobilitätsangeboten ermittelt. Als mobilitätssuffizient galten die 32 Proband/innen aufgrund ihrer - mit einem einwöchigen Wegetagebuch ermittelten - Alltagsmobilität, die zu unterdurchschnittlichen Treibhausgasemissionen im Vergleich zu Personen gleichen Geschlechts und gleicher Lebensphase führte. Die Vergleichsdaten entstammen der Verkehrsbefragung Wuppertal 2011 (Hoppe et al. 2012) und der Studie „Mobilität in Deutschland 2008“ für Kernstädte mit mehr als 100.000 Einwohner/innen (Follmer et al. 2010). Ausgewählt wurden nur Proband/innen, die angaben, hauptsächlich Verkehrsmittel des Umweltverbundes zu nutzen. Sie wohnten in Wuppertal mit guter Nahversorgung und einem guten ÖPNV-Angebot. Trotzdem unterschieden sich die Proband/innen im jeweils genutzten Hauptverkehrsmittel: Fuß (sieben Teilnehmer/innen), Fahrrad (acht Teilnehmer/innen), ÖPNV (zehn Teilnehmer/innen) und Pkw (sieben Teilnehmer/innen). Dabei verfügten 27 Studienteilnehmer/innen über ein ÖPNV-Abo und 20 hatten mindestens einen Pkw im Haushalt zur Verfügung. Auf Nachfrage gab nur eine Teilnehmerin an, aus Kostengründen kein Auto zu besitzen.

\section{Reduktion von Treibhausgasen}

Was kann Mobilitätssuffizienz zur Treibhausgasreduktion beitragen? Abbildung 1 zeigt das in der Fallstudie ermittelte Reduktionspotenzial. Im Durchschnitt erreichten die Proband/innen eine Minderung der Treibhausgase um 63,2\% im Vergleich zu Personen gleichen Geschlechts und gleicher Lebensphase. Selbst die sieben Teilnehmer/innen, die als Hauptverkehrsmittel ein Auto benutzten, schafften im Schnitt eine 41\%-ige Reduktion. Darüber hinaus zeigte das zum Vergleichsmaßstab emissionsniedrigste Probandendrittel, dass die Anforderung des Klimaschutzplans der deutschen Bundesregierung für 2050 im Bereich der Mobilität bereits heute zu erreichen ist. Ohne den Einsatz innovativer Effizienz- und Konsistenztechnik verursachten diese elf Proband/innen um 89,9\% geringere $\mathrm{CO}_{2 \text { eq }}{ }^{-}$ Emissionen allein durch ihr individuelles Konsumverhalten.

Dass die Proband/innen zur Realisierung der Treibhausgasreduktionen nicht etwa zu Hause geblieben sind und auf Mobilität verzichtet haben, zeigt eine detaillierte Betrachtung der drei Handlungsoptionen aus der obigen Definition. In Abbildung 2 bilden die drei Umweltwirkungsparameter zur Berechnung der Treibhausgasemission gleichzeitig die drei Möglichkeiten zum suffizienten Mobilitätskonsum ab. Da die Parameter in der Formel als Multiplikatoren verbunden sind, multipliziert sich die Wirkung bei kombinierter Nutzung. 


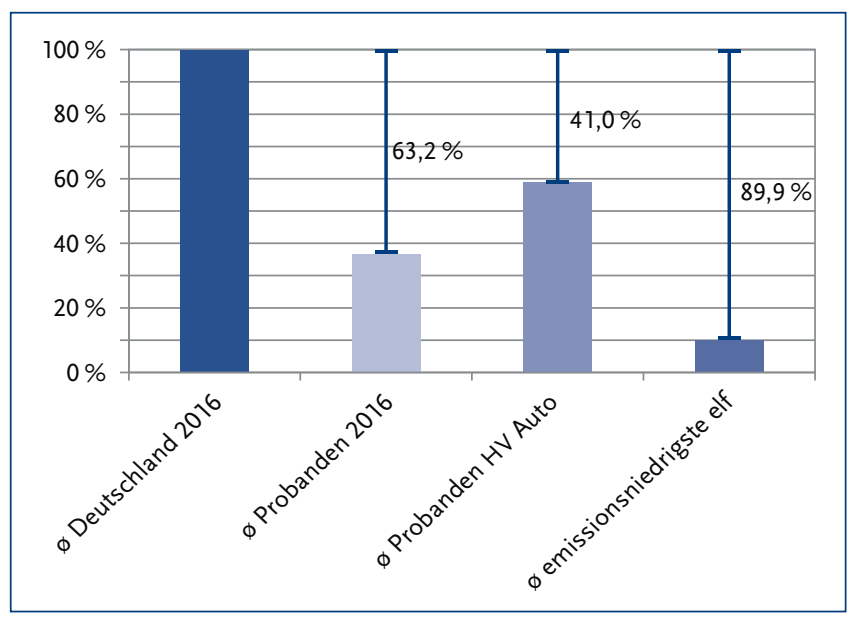

Abbildung 1: Mögliche $\mathrm{CO}_{2 \text { eq }}$-Reduktion durch Mobilitätssuffizienz

(Quelle: nach Follmer et al. 2010; UBA 2016;

o.g. Fallstudie; eigene Berechnung und Darstellung)

Abbildung 3 konkretisiert die Berechnung mit den für eine Probandin erfassten Werten. Der Vergleich mit den Durchschnittswerten von Personen gleichen Geschlechts und gleicher Lebensphase in Deutschland zeigt, dass die Probandin selbst bei fast doppelt so hohen Mobilitätsaktivitäten (183,7\%) den für 2050 geplante Zielkorridor des Klimaschutzplanes erreicht, wenn sie die Wege mit verhaltensbedingt geringeren Treibhausgasemissionen (17,6\%) zurücklegt und diese kurz genug sind $(18,3 \%)$.

\section{Handlungsoptionen}

Insgesamt machen die Proband/innen von der Handlungsoption weniger Wege kaum Gebrauch, sodass sie die dargestellten Treibhausgasreduktionen ohne Minderung von Mobilitätsaktivitäten erreichen.

Dafür wurden die anderen beiden Handlungsoptionen umso konsequenter genutzt. Wie Abbildung 4 zeigt, war der durchschnittliche Probandenweg mit 6,3 Kilometer um 30\% kürzer als sonst in Wuppertal üblich. Dabei kam es nicht zufällig zu diesen kürzeren Wegen. Knapp die Hälfte der Proband/innen integriert gezielt Einkäufe oder Erledigungen in Wegeketten oder in einen Arbeits- oder Freizeitweg. Über ein Drittel der Probanden haben Wohn- oder Arbeitsort bewusst für einen kurzen Weg zur Arbeit und/oder zum Einkaufen gewählt. Von einigen Proband/innen wird der Zeitgewinn als Argument für kurze Wege hervorgehoben.

Die Nutzung der Handlungsoption, Wege mit verhaltensbedingt geringerem Emissionsfaktor zurückzulegen, zeigt $\mathrm{Ab}$ bildung 5. Die Probanden sind 2,6 Mal seltener im Kfz unterwegs als der durchschnittliche Wuppertaler, dafür aber 2,7 Mal so häufig im Umweltverbund. Ein Drittel der Studienteilnehmer gibt die Umweltfreundlichkeit des Verkehrsmittels als wesentliches Entscheidungskriterium an.

\section{Motivation für Mobilitätsentscheidungen}

Welche Motivation führt zur Mobilitätssuffizienz? Nur eine Probandin sagt, sie besitze aus Kostengründen kein Auto, 27 Proband/innen haben ein ÖPNV-Abo. Konsumverweigerung und Geldmangel waren somit nicht als ausschlaggebende Gründe festzustellen.

Stattdessen erklären zwei Drittel der Proband/innen, dass sie mit ihrer Art der Mobilität Lebensqualität gewinnen. Knapp ein Drittel der Proband/innen zieht diese aus dem Genuss ihrer Autofreiheit. Sie sehen ein eigenes Auto als Ballast, und es fehlt ihnen nicht. Zwei Dritteln der Studienteilnehmer/innen bringt die Nutzung des Umweltverbundes Lebensqualität, sie resultiert entweder aus der Bewegung beim Radfahren und $\mathrm{Zu}$ fußgehen oder aus der Freizeitqualität der Wege bei der ÖPNV Nutzung. Dort werden die Probanden chauffiert und können die Zeit nutzen zum Lesen, für Sozialkontakte, Landschaftsbeobachtung und sich „baumeln lassen“.

\begin{tabular}{|c|c|c|c|c|c|c|c|}
\hline & & Umweltwirkungsparameter & & & & & \\
\hline \multirow[t]{2}{*}{ Mobilitätsemission p.a. (in $\mathrm{CO}_{2 \text { eq }}$ ) } & \multirow[t]{2}{*}{$=$} & $\varnothing \mathrm{CO}_{2 \text { eq }}$ je Personenkilometer & $x$ & $\varnothing \mathrm{km}$ je Weg & $x$ & $\varnothing$ Wege pro Tag & 365 Tage \\
\hline & & Emissionsfaktor verringern & & kürzere Wege & & weniger Wege & \\
\hline
\end{tabular}

\begin{tabular}{|c|c|c|c|c|c|c|c|c|}
\hline \multicolumn{9}{|l|}{ Formel } \\
\hline$\varnothing \mathrm{CO}_{2 \text { eq }}$ je Personenkilometer & $\times$ & $\varnothing$ km je Weg & $\times$ & $\varnothing$ Wege pro Tag & $x$ & 365 Tage & $=$ & Mobilitätsemission (in $\mathrm{CO}_{2 \mathrm{eq}}$ p. a.) \\
\hline \multicolumn{9}{|l|}{ Mobilitätsemission der Probandin } \\
\hline $18,7 \mathrm{~g} \mathrm{CO}_{2 \mathrm{eq}} / \mathrm{Pkm}^{2}$ & $x$ & $2,5 \mathrm{~km}$ & $\times$ & 6,4 Wege & $\times$ & 365 Tage & $=$ & 0,11 t $\mathrm{CO}_{2 \text { eq }}$ p.a. \\
\hline \multicolumn{9}{|c|}{ Mobilitätsemission des Vergleichsmaßstabes } \\
\hline $106,4 \mathrm{~g} \mathrm{CO}_{2 \mathrm{eq}} / \mathrm{Pkm}$ & $x$ & $13,6 \mathrm{~km}$ & $\times$ & 3,5 Wege & $x$ & 365 Tage & $=$ & $1,84 \mathrm{t} \mathrm{CO}_{2 \text { eq }}$ p.a. \\
\hline \multicolumn{9}{|c|}{ Nutzung der Handlungsoptionen und Mobilitätsemission der Probandin zum Vergleichswert } \\
\hline $17,6 \%$ & $\times$ & $18,3 \%$ & $\times$ & $183,7 \%$ & $\times$ & & $=$ & $5,9 \% \mathrm{CO}_{2 \text { eq }}$ p.a. \\
\hline
\end{tabular}


Wie kann man den Studienteilnehmer/innen die Mobilitätssuffizienz erleichtern? Während nur 37\% der Deutschen 2010 innerhalb einer Woche mit wechselnden Verkehrsmitteln (Rad, öffentlicher Verkehr und/oder motorisierter Individualverkehr) unterwegs waren (Vortisch et al. 2011, S. 57), waren dies bei den Proband/innen 81\%. Sie entschieden sich situationsspezifisch je nach Ziel, Wetter, Entfernung, zur Verfügung stehender Zeit oder Transportbedarf für das jeweils passende Verkehrsmittel oder deren Kombination. Zur Reduktion des dabei entstehenden Organisationsaufwandes wünscht sich die Hälfte der Proband/innen ein Verkehrsmittel übergreifendes Mobilitätssystem, das vorhandene Angebote räumlich (Umstiegspunkte) und digital (eine App für alles) zu einer integrierten Dienstleistung (Information, Buchung und Abrechnung) verknüpft. Dazu gehört nach Probandenwunsch auch Carsharing mit wohnortnahen Standorten sowie ein System, das Mitfahrgelegenheiten vermittelt. Erst dadurch entsteht eine lückenlose Alternative zum eigenen Auto, welches meist die höchste Treibhausgasemission verursacht.

Mobilitätssuffizienz benötigt Angebote, sei es das beschriebene Mobilitätssystem, einen ausgeprägten ÖPNV oder die Infrastruktur von Rad- und Fußwegen. Als positives Beispiel schafft die Nordbahntrasse, eine im Jahr 2014 eröffnete 23 Kilometer lange Strecke, die abseits des Autoverkehrs für Radfahrer und Fußgänger quer durch Wuppertal führt, Möglichkeiten zur Mobilitätssuffizienz. Auf Grundlage einer repräsentativen Zählung wurde die Nutzung der Nordbahntrasse durch über zwei Millionen Menschen bereits für das Jahr nach ihrer Eröffnung prognostiziert (econex verkehrsconsult GmbH 2015) und die Hälfte der Studienteilnehmer/ innen schätzt ihren Wert für die eigene Selbstbeweglichkeit.

\section{Angebote Ökologisches Wirtschaften}

Die Studie hat gezeigt, dass die Proband/innen zur Realisierung ihrer Mobilitätssuffizienz bereits auf Angebote der Wirtschaft zurückgreifen. Wer einen großen Anteil seiner Mobilität mit dem Rad bewältigt, gibt Geld für dieses aus und kauft Zubehöre wie Fahrradtaschen und (Regen-)Bekleidung. Der hohe Anteil der ÖPNV-Abo-Kunden freut den örtlichen An- bieter durch stabil wiederkehrende monatliche Zahlungen. Für Menschen, die multimodal ohne eigenes Auto mobil sind, wird ein alltägliches Reiseaccessoire wie ein Rucksack zum unabdingbaren, den Kofferraum ersetzenden Bestandteil der Mobilität. So haben ein Drittel der Probanden ihren Rucksack immer dabei und würden ihn bei Ausfall sofort austauschen.

Zusätzliche Angebote für suffizienten Konsum durch Unternehmen können $\mathrm{zu}$ einer sich gegenseitig unterstützenden Ausweitung der Suffizienz führen: Konsumenten erhalten mehr Gelegenheiten zum suffizienten Handeln, nehmen die Angebote an, fördern damit ökologisches Wirtschaften und ermöglichen so die Einhaltung notwendiger Umweltgrenzen. Welche zusätzlichen Angebote benötigten die Probanden? Die durchgeführte Studie zeigt zwei wesentliche Hindernisse der Mobilitätssuffizienz, aus denen unternehmerische Chancen re- 
sultieren können. Derzeit begrenzen notwendige Transporte von Gegenständen und die bereits genannten Beschränkungen der Multimodalität suffizientes Mobilitätsverhalten.

Der notwendige Transport des wöchentlichen Großeinkaufes macht den Proband/innen die Nutzung der Verkehrsmittel des Umweltverbundes schwer. Wurde das Auto auf Strecken ohne Einkäufe nur zu 33,2\% genutzt, stieg dessen Nutzungsanteil bei Einkaufsfahrten auf 47,2\%. Für diesen Zweck stiegen auch Proband/innen ins Auto, die ansonsten als Hauptverkehrsmittel den Umweltverbund nutzten. Hier kann der lokale Einzelhandel den Mobilitätssuffizienten entgegenkommen. Die von den Proband/innen bevorzugten kurzen Wegestrecken bedingen Einkaufsgelegenheiten in der Nähe. So wünschen sich mehr als ein Drittel der Befragten die unmittelbare Bündelung von Angeboten in ihrem Wohnumfeld in Form von Quartiersläden. Dass dieses Konzept aufgeht, zeigt der Wuppertaler CAP Markt, der in einem Stadtteil mit vorher mangelhafter Nahversorgung im ersten vollen Geschäftsjahr 150.000 Kund/innen hatte (CAP Markt, eigene Angabe). Auch das Liefern von täglich Benötigtem bietet weitere Geschäftschancen. Konkurrieren mittlerweile Lieferanten von fertigem Essen um jeden Kunden, so sind Lieferdienste für Waren des täglichen Bedarfs bisher noch schwer zu finden. Dass manche Einkaufsmärkte des REWE- und Edeka-Konzerns auch nach Hause liefern, wird so lange ein Geheimtipp bleiben, bis diese offensiv damit werben. Tatsächlich mangelt es an Plattformen, die die vorhandenen Angebote bündeln und den Zugang für Kund/ innen damit erleichtern. So existiert in Wuppertal zwar das Angebot, Online City Wuppertal', das die taggleiche Lieferung vieler Produkte von örtlichen Händlern ermöglicht, aber ein umfassendes Sortiment eines Supermarktes eben nicht bietet. Mobilitätssuffiziente, die das Einkaufserlebnis im Laden nicht missen wollen, haben ein anderes Problem. Was machen sie mit ihren Einkäufen bei einer größeren Einkaufstour, wenn sie ohne Auto und somit ohne Kofferraum unterwegs sind? Den Proband/innen fehlen für diese Situation Möglichkeiten der Gepäckaufbewahrung. Mehr als ein Drittel der Befragten wünscht sich dort, wo sie einkaufen, Möglichkeiten zur Zwischenlagerung, um ohne Transportlast weiter einzukaufen.

Von fehlenden Möglichkeiten zur Multimodalität wurde bereits oben berichtet. Den Mobilitätssuffizienten mangelt es an der Vernetzung bestehender Beförderungsmöglichkeiten mit der zusätzlichen Integration weiterer Angebote, wie Carsharing und private Mitnahmemöglichkeiten, zu einem umfassenden Mobilitätssystem. Das vor Ort vorhandene Carsharing-Angebot von cambio nutzen die Probanden wegen unzureichender spontaner und örtlicher Verfügbarkeit nur in Ausnahmesituationen. Gewünscht wird eine zuverlässige Verfügbarkeit des Fahrzeugs in einer Entfernung von circa 400 Metern vom Ort des Bedarfs, der meist der Wohnadresse entspricht. Eine solch umfassende Verfügbarkeit von Carsharing Fahrzeugen scheitert laut der cambio-Geschäftsführerin an der dazu notwendigen finanziellen unternehmerischen Vorleistung für die An- schaffung der großen Anzahl dafür benötigter Fahrzeuge (Warmke 2017). Hätte Airbnb sein Geschäftsmodell sukzessive mit eigenen Unterkünften aufgebaut, würden sie heute nur einen sehr geringen Anteil der derzeitigen Umsätze tätigen. Erst die Nutzung vorhandener Wohnungen und deren Vermittlung von privat an privat hat Airbnb stark gemacht. Diesem Modell folgend, könnten eingeführte Carsharing-Anbieter ihr langjährig aufgebautes Vertrauen vor Ort nutzen und das notwendige Angebotspotenzial durch die Vermittlung von Nachbarschaftsautos von privat an privat schaffen. Der von Mobilitätssuffizienten gewünschte Angebotsumfang würde somit ohne finanzielle Investition in zusätzliche Fahrzeuge - und damit wirklich umweltfreundlich - realisiert. Eine Investition in den eigenen Fuhrpark kann der Carsharing-Anbieter somit aufschieben, bis ihm die Auslastung eines neuen Fahrzeuges sicher ist. Das Konzept des sukzessiven Geschäftsausbaus, der zunächst bereits vorhandene Privatautos nutzt, kann auch der ÖPNV-Betreiber vor Ort nutzen. Warum nicht Fahrten, die mit dem ÖPNV-Fuhrpark nicht profitabel zu bedienen sind, durch ein Mitnahmesystem anbieten, das private Autofahrten nutzt und Fahrer/innen und Mitfahrer/innen mit einem ähnlichen Streckenwunsch zusammenbringt? Beispiele wie „Taxito“, „Garantiert mobil“ und „Mobilfalt" gibt es bereits. Ein Mobilitätssystem, das die heutigen Möglichkeiten des ÖPNV, ein ausgeweitetes Carsharing und das Angebot privater Mitnahmen integriert, braucht für die von den Mobilitätssuffizienten gewünschte Multimodalität, die eine Mobilitätskette ähnlich dem Automobil bietet, nur noch eine digitale Mobilitätsplattform und Orte des Übergangs von einem zum anderen Beförderungsmittel. Dabei hätte die Mehrheit der Befragten auch nichts dagegen, zukünftig in autonomen Fahrzeugen befördert zu werden. Schon das derzeitige hohe Maß an Multimodalität, das stark zur Mobilitätssuffizienz beiträgt, wird von fast allen Probanden nur durch die Nutzung des digitalen Routings via App erreicht. So wundert deren Erwartung an das zukünftige vernetzte Mobilitätssystem nicht: Es solle bitte Anbieter übergreifend Zugang, Routing, Bezahlung und lückenlosen Verkehrsmittelübergang in einem digitalen Angebot bündeln. Durch ihre Bekanntheit und das bereits bestehende Mobilitätsangebot vor Ort sind für das beschriebene vernetzte System sicherlich die Betreiber des öffentlichen Nahverkehrs prädestiniert. Deren Interesse liegt bisher aber weniger im beschriebenen individuellen Kollektivverkehr als in der althergebrachten Dienstleistung, Passagiere in möglichst großen Gefäßen zu festgelegten Zeiten auf festgelegten Routen zu befördern.

\section{Fazit}

Das ermutigende Ergebnis der Studie ist, dass Konsumreduktion oder -verlagerung auch im Bereich der Mobilität sofort - ohne weiteres Warten auf technische Innovationen - zur Reduktion des Umweltverbrauches führen kann. So kamen die Probanden allein durch ihr suffizientes Mobilitätsverhalten zu weit unterdurchschnittlichen Treibhausgasemissionen. Dabei 
benötigt suffizientes Verhalten im untersuchten Bereich der Alltagsmobilität keinen Aktivitätsverzicht und kann einen $\mathrm{Zu}$ wachs an Lebensqualität mit sich bringen, der nach Linz (ebd. 2002, S. 13) einen gesellschaftlichen Wandel zu mehr Suffizienz erst ermöglicht. Dazu braucht es auch von Unternehmensseite passende Angebote, die die Nachfrage der Mobilitätssuffizienten gezielt bedienen und damit mehr Suffizienz ermöglichen.

Es ist an der Zeit, als Beitrag zur Lösung von Umweltproblemen mehr Suffizienz zu nutzen. Dazu benötigt es weitergehende Forschung, die den nicht repräsentativen Geltungsbereich der vorgestellten Ergebnisse auf breiterer Datengrundlage absichert und weitere Konsumbereiche betrachtet.

\section{Anmerkung}

Ausführliche Informationen liefert das soeben erschienene Buch: Profijt, M.: Mobilitätssuffizienz. Grundlagen - Messung - Förderung. oekom. ISBN 978-3-96238-070-0

\section{Literatur}

Bundesministerium für Verkehr, Bau und Reaktorsicherheit (BMUB) (2016): Klimaschutzplan 2050. Klimaschutzpolitische Grundsätze und Ziele der Bundesregierung. Berlin.

econex verkehrsconsult GmbH (Hrsg.) (2015): Nutzererhebung Nordbahntrasse. Materialien Nordbahntrasse. Wuppertal.

Follmer, R. et al. (2010) (im Auftrag des Bundesministerium für Verkehr, Bau und Stadtentwicklung (BMUB)): Mobilität in Deutschland 2008. Ergebnisbericht. Struktur - Aufkommen - Emissionen - Trends. Bonn, infas Institut für angewandte Sozialwissenschaft.

Hoppe, R./Woschei, K. (2012): Verkehrsbefragung 2011. Stadt Wuppertal. Bericht Verkehrsbefragung zum werktäglichen Verkehrsverhalten der Bevölkerung in Wuppertal 2011. Wuppertal, Planungsgesellschaft Verkehr Köln.

Knörr, W./Heidt, C./Gores, S./Bergk, F. (2016) (im Auftrag des Umweltbundesamts (UBA)): Aktualisierung Daten- und Rechenmodell: Energieverbrauch und Schafstoffemissionen des motorisierten Verkehrs in Deutschland 1960-2035. (TREMOD) für die Emissionsberichterstattung 2016. Heidelberg, Ifeu-Institut für Energie- und Umweltforschung.
Lambrecht, U./Helms, H./Dünnebeil, F. (2013): Steigende Umweltanforderungen. Was bedeutet dies für den Verkehr? In: Beckmann, K./KleinHitpaß, A. (Hrsg.): Nicht weniger unterwegs, sondern intelligenter? Neue Mobilitätskonzepte. Berlin, Deutsches Institut für Urbanistik, S. 59-77.

Linz, M. (2002): Warum Suffizienz unentbehrlich ist. In: Wuppertal Institut für Klima, Umwelt, Energie GmbH (Hrsg.): Von nichts zu viel: Suffizienz gehört zur Zukunftsfähigkeit. Wuppertal: Wuppertal Institut für Klima, Umwelt und Energie, S. 7-14.

Umweltbundesamt (UBA) (2017): Klimabilanz 2016: Verkehr und kühle Witterung lassen Emissionen steigen. www.umweltbundesamt.de/sites/ default/files/medien/479/dokumente/pm-2017-o9_thgnahzeitprognose_2016.pdf

Warmke, G. (2017): CarSharing in Wuppertal. So viel Auto macht Sinn. In: Bergische Universität Wuppertal, Zentrum für Transformationsforschung und Nachhaltigkeit, Veranstaltungsreihe Mobilität und Verkehr 16. 05. 2017.

Vortisch, P./Zumkeller, D./Kagerbauer, M./Streit, T./Chlond, B./Wirtz, M. (2011) (im Auftrag des Bundeministerium für Verkehr, Bau- und Stadtentwicklung (BMVI)): Deutsches Mobilitätspanel (MOP) wissenschaftliche Begleitung und erste Auswertung. Bericht 2011/2012: Alltagsmobilität\& Tankbuch. Karlsruhe, Karlsruher Institut für Technologie.

\section{AUTOR + KONTAKT}

Markus Profijt studierte Betriebswirtschaftslehre und Umweltwissenschaften und promovierte als externer Doktorand im Forschungsverbund des Wuppertal Instituts mit der Bergischen Universität Wuppertal am Fachzentrum Verkehr zum Doktor der Ingenieurwissenschaften.

Bergische Universität Wuppertal, Fachzentrum Verkehr, Pauluskirchstraße 7, 42285 Wuppertal. Tel.: +49 202 439-4088. E-Mail: markus.profijt@uni-wuppertal.de
Nachhaltigkeit

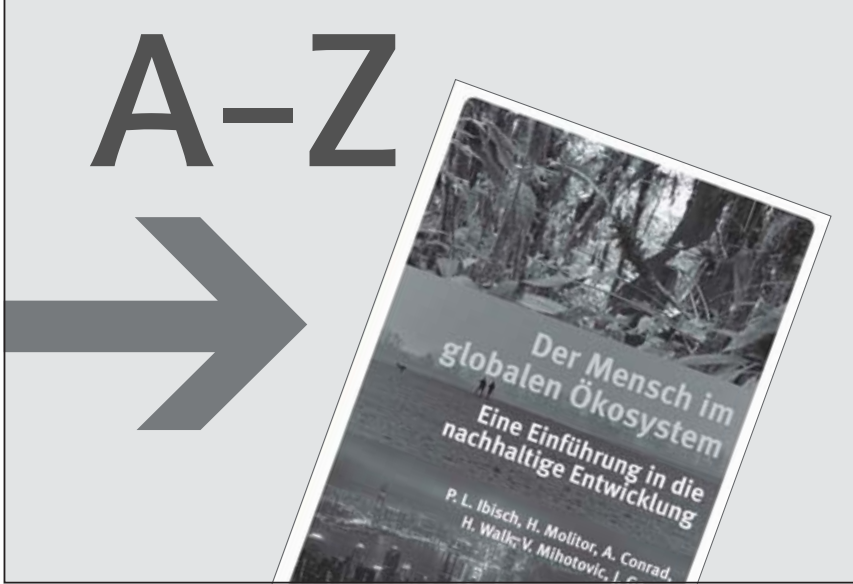

\section{wie Transformationswerkzeug}

Dieses fachübergreifende Lehrbuch für Studierende und Studieninteressierte gibt einen umfassenden Überblick zur nachhaltigen Entwicklung - und bietet darüber hinaus neue Vorschläge zu deren weiterer Ausgestaltung. Von einem interdisziplinären Autorenteam verfasst, spannt es einen Bogen von der "Erfindung" der Nachhaltigkeit bis hin zu aktuellen Ansätzen politischer und ökonomischer Transformation. Es richtet sich auch an interessierte Laien und alle "Transformateure", die sich für eine nachhaltige Entwicklung einsetzen.

P. L. Ibisch, H. Molitor, A. Conrad, H. Walk, V. Mihotovic, J. Geyer Der Mensch im globalen Ökosystem

Eine Einführung in die nachhaltige Entwicklung

416 Seiten, broschiert, vierfarbig, mit zahlreichen Abbildungen, 29,- Euro, ISBN 978-3-96238-01 1-3

Erhältlich im Buchhandel oder versandkostenfre innerhalb Deutschlands bestellbar unter www.oekom.de

Die guten Seiten der Zukunft 